\title{
0 uso de psicofármacos por crianças e adolescentes em um Centro de Atenção Psicossocial Infantil
}

\author{
The use of psychotropic drugs in children and \\ adolescents in a Child Psychosocial Care Center
}

Recebido em: 06/05/2019 Aceito em: $\quad 14 / 09 / 2019$
Orfila Rafaela Trindade da SILVA; Michele Marinho da SILVEIRA Escola de Saúde, Curso de Psicologia. Faculdade Meridional, IMED. Rua Senador Pinheiro, 304, Vila Rodrigues, CEP 99070220. Passo Fundo, RS, Brasil.

E-mail: michele.msilveira@gmail.com

\section{ABSTRACT}

In the last decades, there has been a significant increase in children and adolescents undergoing psychopharmacological treatment. There is a growing number of medico-pedagogical intervention measures, based on the proliferation of diagnoses and drug prescriptions for this portion of the population. The objective was to verify the use of psychotropic drugs by children and adolescents from a Child Psychosocial Care Center (CAPSi). The study was conducted through a retrospective, and cross-sectional survey, with data from 319 records of a CAPSi from the northern region of Rio Grande do Sul, Brazil, from January 2016 to December 2017. It was observed that $66.7 \%$ of the children and adolescents who attended CAPSi used psychotropic drugs, among them the most prescribed were the antipsychotic risperidone with $36.3 \%$ and the stimulant methylphenidate $15.8 \%$. Most drug users were $44.5 \%$ male, with a higher prevalence of attention deficit hyperactivity disorder $17.9 \%$ and depressive symptoms $8.5 \%$, referred by the Municipal Specialized Care Center 27.3\% and Guardianship Council 16.6\%. This study revealed a high use of psychotropic drugs and, consequently, drugs used with diagnostic hypotheses or without a diagnosis, treating symptoms arising from school and family demand.

Keywords: psychotropic drugs; medicalization; child; adolescent.

\section{RESUMO}

Nas últimas décadas houve um aumento significativo de crianças e adolescentes submetidas a tratamento psicofarmacológico. Há um crescente número de medidas interventivas médico-pedagógicas, a partir da proliferação de diagnósticos e prescrições de medicamentos para esta parcela da população. Assim, o objetivo foi verificar a utilização de psicofármacos por crianças e adolescentes de um Centro de Atenção Psicossocial Infantil (CAPSi), por meio de uma pesquisa retrospectiva e transversal, com dados de 319 prontuários de um CAPSi da região norte do Rio Grande do Sul, no período de janeiro de 2016 a dezembro de 2017. Entre as crianças e adolescentes que frequentavam o CAPSi 66,7\% faziam uso de psicofármacos, entre eles, os mais prescritos foram o antipsicótico risperidona com $36,3 \%$ e o estimulante metilfenidato 15,8\%. A maioria dos usuários de psicofármacos era do sexo masculino (44,5\%), com maior prevalência de transtorno de déficit de atenção e hiperatividade (17,9\%) e sintomas depressivos $(8,5 \%)$, encaminhados pelo Centro Municipal de Atendimento Especializado (27,3\%) e Conselho Tutelar )16,6\%). Portanto, este estudo revelou um uso elevado de psicofármacos e, consequentemente, medicamentos usados com hipóteses diagnósticas ou sem um diagnóstico, tratando sintomas advindos de demanda escolar e familiar.

Palavras-chaves: psicotrópicos; medicalização; criança; adolescente. 


\section{INTRODUÇÃO}

No Brasil, por meio do Art. $2^{\circ}$ da lei 8.069/90 do Estatuto da Criança e do Adolescente (ECA), criança é definido como a pessoa com até doze anos de idade incompletos, e adolescente, aquela entre doze e dezoito anos de idade (1). Para a Organização Mundial da Saúde (OMS) os limites cronológicos da adolescência são definidos entre 10 e 19 anos (2).

A população infantil é uma parcela significativa dos usuários dos serviços de saúde mental; o poder público tem sido mais eficiente e, apenas com o atual progresso tecnológico e geográfico, os cuidados com a população infanto-juvenil relativos à educação e saúde tiveram mais destaque (3). Diante disso, pode ser ressaltada a criação, por meio do poder público, do Centro de Atenção Psicossocial Infantil (CAPSi), focado no planejamento e auxílio da saúde mental infanto-juvenil (4).

Alguns estudos $(5,6,7)$ revelaram que a medicalização se transfigura claramente no campo da saúde mental, mais especificamente, nos serviços de saúde. Nesses, é possível observar a indicação demasiada de medicações para sofrimentos de origem mental que, muitas vezes, estão interligados a questões sociais e econômicas. Tal questão retrata um tratamento aplicado apenas no uso de psicofármacos, culminando em uma linha de comunicação deficiente entre profissionais e usuários.

Os psicofármacos são substâncias usadas no tratamento de transtornos mentais, que podem alterar o comportamento e o humor, atuando no sistema nervoso central e estão divididos nas seguintes classes: ansiolíticos, hipnóticos, antidepressivos, antipsicóticos, estabilizadores do humor e psicoestimulantes (8). Estes medicamentos são prescritos a partir das características, das necessidades do paciente e do seu histórico clínico, traçando um plano terapêutico que leva em conta a necessidade do uso de um ou mais medicamentos e, também do poder aquisitivo do usuário (9).

Neste sentido, outros autores $(10,11)$ revelaram que a utilização destes medicamentos necessita de um diagnóstico médico e que devem ser utilizados de modo sensato, tendo em conta que podem ocasionar efeitos e reações adversas, além de dependência e prejuízo ao organismo. Esses me- dicamentos são utilizados para a estabilização dos sintomas ou até mesmo a cura (12).

A grande demanda por diagnósticos e opções de tratamentos que reduzem e facilitam a administração das ânsias do período infanto-juvenil, gera um número exacerbado e relativamente alto de medicamentos usados nesta fase (12). Uma pesquisa sobre a saúde infanto-juvenil brasileira mostrou a introdução tardia da saúde mental em nível nacional e internacional com todas as possíveis patologias dessa fase, bem como os transtornos relativos ao desenvolvimento (13). Para os autores, o que torna relativamente complexo o diagnóstico de transtornos, tanto na infância como na adolescência, é a variação do aparecimento dos sintomas que podem não se encaixar especificamente em uma destas fases (13). Nesta população, a formulação de um diagnóstico de qualidade exige procedimentos de avaliação específicos que incluem, além das próprias crianças e adolescentes, o recurso e as fontes de informação diversas, como familiares, responsáveis, professores, entre outros.

Neste sentido, em um mapeamento para identificar as prováveis demandas que levavam os jovens ao atendimento psiquiátrico, foi constatado que as principais reclamações eram relativas a problemas escolares e de desobediência (14). Para tanto, o objetivo desta pesquisa foi verificar o uso de psicofármacos por crianças e adolescentes atendidas por um Centro de Atenção Psicossocial Infantil (CAPSi) no Rio Grande do Sul, Brasil, com a finalidade de identificar quais eram as medicações mais prescritas e quais as patologias prevalentes nesta população.

\section{MÉTODO}

A presente pesquisa foi desenvolvida a partir de um estudo retrospectivo transversal, com análise documental. Foram utilizados dados secundários provenientes de 319 prontuários de acompanhamento de crianças e adolescentes cadastrados entre janeiro de 2016 a dezembro de 2017 no CAPSi de um município da região norte do Rio Grande do Sul, Brasil.

$\mathrm{Na}$ pesquisa, foram incluídos prontuários de meninas e meninos com idade até 17 anos, sendo 
que não foram incluídos prontuários dos participantes que apresentaram idade maior ou igual a 18 anos, bem como os prontuários em que os dados não estavam completamente preenchidos. Ao todo foram localizados 336 prontuários, que com a exclusão de 17, permaneceu uma amostra de 319 prontuários.

Para este estudo, foi utilizada uma ficha de coleta de dados, na qual constava o sexo, idade, diagnóstico médico, qual ou quais medicamentos psicofármacos fazia uso, quem encaminhou ao CAPSi, por qual motivo, se realizava psicoterapia ou participava de algum grupo terapêutico e se já teve alguma internação.

Como procedimentos éticos e de coleta dos dados, inicialmente, foi solicitada a autorização desta pesquisa na Secretaria de Saúde do requerido município para coletar os dados no CAPSi, após foi encaminhada ao Comitê de Ética e Pesquisa (CEP). Seguidamente a sua aprovação no CEP (CAAE: 83971718.7.0000.5319), foram agendados os dias para a coleta de dados no CAPSi, por meio dos prontuários das crianças e adolescentes. A secretária do local repassou os prontuários de 2016 e 2017 para coletar as informações dos pacientes.

Os dados foram analisados pelo programa Statistical Package for the Social Sciences (SPSS) para Windows, versão 23.0. As variáveis numéricas estão descritas como média \pm desvio padrão e as variáveis qualitativas estão descritas como frequência absoluta e relativa.

\section{RESULTADOS E DISCUSSÃO}

A população atendida pelo CAPSi foi composta por $64,9 \%$ pacientes do sexo masculino e $35,1 \%$ do sexo feminino, com a média de idade de $11,5 \pm$ 3,8 anos, com variação de idade entre 2 e 17 anos conforme apresentado na Tabela 1.

A maioria dos pacientes atendidos no CAPSi (27,3\%) foi encaminhada pelo Centro Municipal de Atendimento Especializado (CEMAE), que atende alunos em situação de vulnerabilidade social. O Conselho Tutelar, órgão de proteção à criança e adolescente, encaminhou $16,6 \%$ dos pacientes, como mostra a Tabela 2.
Das patologias mais frequentes encontradas no serviço, 17,9\% apresentaram como hipótese diagnóstica o Transtorno do Déficit de Atenção com Hiperatividade (TDAH), visto que a grande maioria provinha do CEMAE, que auxilia nas demandas escolares. Diante disso, foi possível observar que praticamente metade dos diagnósticos obtidos tem pouca prevalência, no máximo um paciente, gerando um percentual de $0,3 \%$, e, que, $25,7 \%$ dos pacientes não tinham hipótese diagnóstica registrada no prontuário (Tabela 3 ).

Tabela 1. Distribuição do número de crianças e adolescentes, de acordo com o gênero e idade, acompanhadas emCentro de Atenção Psicossocial Infantil (CAPSi) do RS (janeiro de 2016-dezembro de 2017)

\begin{tabular}{|l|l|l|}
\hline Variável & N & $\%$ \\
\hline Masculino & 207 & 64,9 \\
\hline Feminino & 112 & 35,1 \\
\hline Idade (anos) & & \\
\hline 17 & 38 & 11,9 \\
\hline 14 & 33 & 10,3 \\
\hline 16 & 28 & 8,8 \\
\hline 8 & 27 & 8,5 \\
\hline 13 & 27 & 8,5 \\
\hline 7 & 24 & 7,5 \\
\hline 11 & 24 & 7,5 \\
\hline 15 & 23 & 7,2 \\
\hline 9 & 22 & 6,9 \\
\hline 10 & 20 & 6,3 \\
\hline 12 & 19 & 6 \\
\hline 6 & 16 & 5 \\
\hline 5 & 11 & 3,5 \\
\hline 3 & 3 & 0,9 \\
\hline 4 & 3 & 0,9 \\
\hline 2 & 1 & 0,3 \\
\hline & & \\
\hline 14 & 23 & \\
\hline
\end{tabular}

Os dados revelaram que o sexo masculino foi predominante na utilização de psicofármacos, com 142 casos $(44,5 \%)$, enquanto o sexo feminino apresentou 71 casos $(22,2 \%)$ e a soma desses números gera um total de $213(66,7 \%)$ de crianças e adolescentes utilizando psicofármacos. Destes, o que foi mais prescrito aos pacientes foi o antipsicóticorisperidona, receitado para cerca de 116 pacientes $(36,3 \%)$, seguido do estimulante metilfenidato para 
$48(15,8 \%)$, prescrito para TDAH, e o antidepressivo fluoxetina, para $44(13,7 \%)$, prescrito para ansiedade e sintomas depressivos conforme a Tabela 4. Além disso, muitos faziam o uso combinado de mais medicamentos.

Outras informações relevantes deste estudo revelaram que $2,1 \%$ pacientes haviam sido submetidos anteriormente à internação compulsória. Ainda, das crianças e adolescentes, 169 (53,0\%) realizavam arteterapia, $93(29,2 \%)$ sem participação concomitante com outras atividades no CAPSi, $21(6,6 \%)$ estavam em grupo psicopedagógico e 10 (3,1\%) em grupo terapêutico.

Pesquisas sobre a medicalização na infância mostraram dados que preocupam e requerem uma reflexão acerca do uso de psicofármacos de maneira significativa nessa população (15-19). Um estudo sobre crianças e adolescentes atendidas em um CAPSi da região Nordeste do Brasil, revelou que a maioria também era do sexo masculino com $74,2 \%$ (20). Em meio à população infantil usuária de psicofármacos, a maior parte era do sexo masculino, frequentava creches ou ensino fundamental e fazia uso de medicamentos para transtorno de déficit de atenção e hiperatividade, sintomas de ansiedade e transtornos de aprendizagem (3). Tais números são coerentes com os encontrados neste estudo, com mais da metade dos pacientes atendidos usando medicamentos (213 ou $66,7 \%$ ), do sexo masculino $(44,5 \%)$ e com hipótese diagnóstica de transtorno de déficit de atenção e hiperatividade $(17,9 \%)$.

Tabela 2. Distribuição do número de crianças e adolescentes, de acordo com o encaminhamento, acompanhadas em Centro de Atenção Psicossocial Infantil (CAPSi) do RS (janeiro de 2016-dezembro de 2017)

\begin{tabular}{|c|c|c|}
\hline Variável & $\mathbf{N}$ & $\%$ \\
\hline CEMAE (Centro Municipal de Atendimento Especializado) & 87 & 27,3 \\
\hline Conselho Tutelar & 53 & 16,6 \\
\hline Escola & 35 & 11 \\
\hline Sem Informação & 28 & 8,8 \\
\hline Demanda Espontânea & 26 & 8,2 \\
\hline Hospitais & 14 & 4,4 \\
\hline CAIS (Centro de Assistência Integral a Saúde) & 13 & 4,1 \\
\hline UBS (Unidade Básica de Saúde) & 12 & 3,8 \\
\hline CREAS (Centro de Referência Especializado de Assistência Social) & 11 & 3,5 \\
\hline CAPS (Centro de Atenção Psicossocial) & 9 & 2,8 \\
\hline CRAS (Centro de Referência de Assistência Social) & 4 & 1,3 \\
\hline Psiquiatra & 4 & 1,3 \\
\hline APAE (Associação de Pais e Amigos dos Excepcionais) & 3 & 0,9 \\
\hline Ambulatório & 3 & 0,9 \\
\hline PSF (Programa Saúde da Família) & 3 & 0,9 \\
\hline Ordem Judicial & 3 & 0,9 \\
\hline Fonoaudiólogo & 2 & 0,6 \\
\hline Neurologista & 2 & 0,6 \\
\hline CEPIA (Centro de Estudos e Proteção à Infância e à Adolescência) & 1 & 0,3 \\
\hline CIAE (Centro Integrado de Atendimento ao Estudante) & 1 & 0,3 \\
\hline SAE (Sistematização da Assistência à Enfermagem) & 1 & 0,3 \\
\hline Assistente Social & 1 & 0,3 \\
\hline SEMCAS (Secretaria de Cidadania e Assistência Social) & 1 & 0,3 \\
\hline Defensoria pública & 1 & 0,3 \\
\hline Casa de Acolhimento & 1 & 0,3 \\
\hline
\end{tabular}


O contexto social em que as crianças encaminhadas por queixa comportamental estão inseridas é significativo na percepção das possíveis patologias apresentadas (21). Os dados expostos na Tabela 2 apresentam altos índices de encaminhamento por parte do Centro Municipal de Atendimento Especializado e do Conselho Tutelar (43,9\%), evidenciando uma desestrutura familiar.

Há um número evidente de encaminhamentos por parte das escolas ou das instituições ligadas a elas por queixas comportamentais em que, muitas vezes, não seria necessário o emprego de medicações e sim, encaminhamentos para oficinas e/ou terapias (21). Pode ser observado que a medicalização tem se expressado na transformação de problemas educacionais, advindos de uma escola que historicamente produz fracasso escolar, em doenças e transtornos que têm de ser resolvidos pelos profissionais de saúde, com destaque para a medicina. Cria-se, assim, uma demanda por esses profissionais, gerando uma cultura de encaminhamento nas instituições escolares (22).

Com isso, foi mostrado o quanto se tornou comum para as escolas e famílias o controle do comportamento das crianças por meio do ato da medicalização, o que torna a relação padronizada e com baixo investimento nos quesitos emocionais e éticos. A utilização de medicamentos vem sendo amplamente disseminada nos meios familiares e escolares, mas os profissionais da área da saúde pública devem buscar alternativas (23).

A utilização ou não de psicofármacos está relacionada com a sintomatologia apresentada pelo paciente, que deve ser avaliada criteriosamente por um profissional, com o propósito de formular um possível diagnóstico. Os medicamentos são os tratamentos preferenciais para múltiplos transtornos, como os de origem bipolar ou esquizofrênica, em depressões graves ou de ataques de pânico. Em outros, como fobias, e transtornos de personalidade, ou até mesmo em questões situacionais; as psicoterapias podem e devem ser preferenciais sendo que, em muitas circunstâncias a associação dos dois métodos é eficaz (24).

Além do tratamento medicamentoso, outras modalidades de atividades eram realizadas pelas crianças e adolescentes, em que 169 (53,0\%) frequentavam arteterapia, $21(6,6 \%)$ grupo psicopeda- gógico e $10(3,1 \%)$ grupo terapêutico. Entretanto, algumas crianças e adolescentes só faziam o uso de medicamentos sem a participação de um acompanhamento em oficinas na instituição. A partir disso, a medicação tornou-se, literalmente, o remédio para os problemas de toda ordem, seja de ordem médica ou não, e dela se utiliza para compreender e solucionar os conflitos produzidos socialmente, tentando, mediante intervenções voltadas para a criança em particular, resoluções para os problemas da educação, da saúde, da família e da relação entre adultos e crianças em geral. Assim, muitas condutas, como a indisciplina ou a não aprendizagem, que, a nosso juízo, evidenciam os precários resultados do sistema educacional e escolar em nossos dias, possuem uma classificação e são diagnosticadas segundo os critérios do DSM-IV ou do CID-10. O CAPSi não está excluído desse processo, ao contrário, participa de forma ativa: constitui a via para a obtenção desse diagnóstico e, em consequência, da medicação (25).

Foi observado um aumento do número de diagnósticos médicos e de subsequente ingestão de medicação por crianças e jovens em idade escolar. Neste contexto, a principal patologia prevalente nas salas de aula das escolas foi o Transtorno do Déficit de Atenção com Hiperatividade (26), corroborando os achados deste estudo, que apresentaram o TDAH com $17,9 \%$ de prevalência e o que apresentou mais necessidade de medicamentos.

A medicalização infantil está sendo vista como um processo por meio do qual alguns aspectos da vida humana, como a inquietude e a desobediência infanto-juvenil sejam considerados "problemas médicos". Anteriormente, estes indicativos não eram entendidos como patológicos e isso parece estar atingindo proporções alarmantes em muitos países, como o Brasil, os Estados Unidos, o Reino Unido e Portugal (18).

Dessa forma, o processo de medicalização aumentou significativamente e atinge praticamente todas as fases da vida. Por esta lógica, o sujeito possuidor de uma divergência humana é visto como portador de uma doença. Assim, a medicalização tem sido utilizada pelos médicos para as dificuldades de aprendizagem das crianças, transformando estas em patologias (16). 
De acordo com a Organização das Nações Unidas (ONU), um dos psicofármacos prescritos de forma mais indiscriminada na atualidade é o metilfenidato, consumido de forma abundante nos Estados Unidos e um dos mais receitados atualmente nos consultórios brasileiros para crianças e adolescentes, pacientes portadores de TDAH (27). Junta- mente com a risperidona o metilfenidato é um dos medicamentos mais receitados conforme dados observados na Tabela 4, em que $116(36,3 \%)$ faziam uso de risperidona e $48(15,8 \%)$ de metilfenidato.

Em muitas das prescrições para TDAH a risperidona e o metilfenidato eram utilizadas em conjunto.

Tabela 3. Distribuição das hipóteses diagnósticas das crianças e acompanhadas emCentro de Atenção Psicossocial Infantil (CAPSi) do RS (janeiro de 2016-dezembro de 2017)

\begin{tabular}{|c|c|c|c|}
\hline CID* & Hipótese & N & $\%$ \\
\hline -ーーーー & Sem hipótese diagnóstica & 82 & 25,7 \\
\hline CID 10 F90 & TDAH & 57 & 17,9 \\
\hline CID 10 F32 & Transtorno Depressivo & 27 & 8,5 \\
\hline CID 10 F84.1 & Transtorno do EspectroAutista & 26 & 8,2 \\
\hline CID10 F70 & Retardo Mental Leve & 23 & 7,2 \\
\hline CID 10 F19 & DependênciaQuímica & 18 & 5,6 \\
\hline CID10 F91.3 & TOD (Transtorno de Oposição Desafiante) & 17 & 5,3 \\
\hline CID 10 F31 & TranstornoAfetivo Bipolar & 7 & 2,2 \\
\hline CID 10 F63 & Transtornos dos hábitos e dos impulsos & 7 & 2,2 \\
\hline CID 10 F60.3 & Transtorno de personalidade Borderline & 6 & 1,9 \\
\hline CID 10 F91.9 & Transtorno de Conduta & 5 & 1,6 \\
\hline CID 10 F20 & Esquizofrenia & 5 & 1,6 \\
\hline CID 10 F84 & TranstornosGlobais do desenvolvimento & 5 & 1,6 \\
\hline CID 10 F06.7 & Transtornocognitivoleve & 5 & 1,6 \\
\hline CID 10 F41.2 & Transtorno misto ansioso e depressivo & 4 & 1,3 \\
\hline F00 F99 & TranstornosMentais e Comportamentais & 4 & 1,3 \\
\hline CID 10 F 84.5 & Síndrome de Aspenger & 3 & 0,9 \\
\hline CID10 F30/F39 & Transtorno de Humor & 2 & 0,6 \\
\hline CID 10 F41.9 & Transtornoansiosonãoespecificado & 1 & 0,3 \\
\hline X60 X84 & Tentativa de suicídio & 1 & 0,3 \\
\hline CID 10 P21 & Anoxia Neonatal & 1 & 0,3 \\
\hline CID 10 F12 & Transtorno Devido ao uso de Canabioides & 1 & 0,3 \\
\hline CID 10 F30/F39 & Humor Deprimido & 1 & 0,3 \\
\hline CID 10 F81 & Transtorno de Aprendizagem & 1 & 0,3 \\
\hline CID 10 R48 & Dislexia & 1 & 0,3 \\
\hline CID 10 Z 63.4 & Luto Normal & 1 & 0,3 \\
\hline CID 10 F 91.3 & Distúrbio desafiador e de oposição & 1 & 0,3 \\
\hline CID 10 F 41.0 & Transtorno do Pânico & 1 & 0,3 \\
\hline CID10 F43.1 & Transtorno de Estresse pós Traumático & 1 & 0,3 \\
\hline CID 10 F63.8 & TranstornoExplosivoIntermitente & 1 & 0,3 \\
\hline CID 10 F60.1 & Personalidadeesquizóide & 1 & 0,3 \\
\hline CID 10 F94, 0 & MutismoSeletivo & 1 & 0,3 \\
\hline CID 10 G40 & Epilepsia & 1 & 0,3 \\
\hline CID $10 Q 90.9$ & Síndrome de Down & 1 & 0,3 \\
\hline
\end{tabular}

*Código de Classificação Internacional de Doenças. 
Em outro estudo no CAPSi de Fortaleza, Estado do Ceará, os medicamentos mais prescritos eram os antipsicóticos, em especial a risperidona (28), corroborando aos achados desta pesquisa. É importante mencionar que efeitos adversos de psicofármacos em crianças e adolescentes podem causar o agravamento de depressão e tentativas de suicídio, síndrome neuroléptica maligna, efeitos extrapiramidais, problemas metabólicos e cardiovasculares como obesidade e risco aumentado de diabetes (29).

Os psicofármacos mais requisitados são ansiolíticos e hipnóticos, muito utilizados nos transtornos de ansiedade, entre eles síndrome do pânico ou agorafobia, além dos antidepressivos para transtorno depressivo e de ansiedade (30). Neste caso, na Tabela 4 estão apresentadas evidências de que 44 dos indivíduos faziam uso de fluoxetina, devido a sintomas depressivos.

Pode ser considerado que o uso dos medicamentos citados vem sendo discutido devido a sua alta taxa de reações adversas apresentadas pelos usuários assíduos. Quando se fala em crianças e adolescentes, deve-se pensar nos efeitos, citados anteriormente, que os medicamentos podem causar a curto e longo prazo nesses indivíduos (31).

Em vista disso, psicofármacos são indicados para pacientes que sofrem de transtornos mentais e devem ser orientados por meio do Sistema Único de Saúde (SUS) no programa Centro de Atenção Psicossocial (CAPS). Neste caso, pode ser citado o CAPSi implementado em municípios acima de 100.000 habitantes, nos quais são oferecidos atendimentos psiquiátrico, neurológico, de enfermagem entre outros (32). O CAPSi foi criado pelo governo com o intuito de tratar e reinserir os portadores de doenças mentais na sociedade, de forma humanizada. Esse trabalho é realizado por meio de uma equipe multiprofissional, possibilitando um ambiente que favoreça e atue de forma terapêutica para o bem-estar dos seus usuários, e, também, atua estendendo os serviços do CAPS aos familiares de seus usuários (32).

Tabela 4. Distribuição do número de crianças e adolescentes, de acordo com a prescrição de psicofármacos, acompanhadas em Centro deAtenção Psicossocial Infantil(CAPSi) do RS (janeiro de 2016-dezembro de 2017)

\begin{tabular}{|c|c|c|c|}
\hline Tipos & Nome do medicamento & $\mathbf{N}$ & $\%$ \\
\hline \multirow{3}{*}{ Ansiolíticos } & Alprazolan & 1 & 0,3 \\
\hline & Cloridrato de Clorpromazina & 4 & 1,2 \\
\hline & Clonazepam & 8 & 2,5 \\
\hline \multirow{6}{*}{ Antidepressivos } & Sertralina & 1 & 0,3 \\
\hline & Imipramina & 37 & 11,5 \\
\hline & Cloridrato de fluoxetina & 44 & 13,7 \\
\hline & 0xalato de Escitalopram & 2 & 0,6 \\
\hline & Cloridrato de nortriptilina & 7 & 2,1 \\
\hline & Cloridrato de Amitriptilina & 3 & 0,9 \\
\hline \multirow{2}{*}{$\begin{array}{l}\text { Estabilizadores de Humor/ } \\
\text { Anticonvulsivante }\end{array}$} & Carbamazepina & 16 & 5,0 \\
\hline & Valproato de Sódio & 1 & 0,3 \\
\hline Estabilizador de Humor & Carbonato de Lítio & 9 & 2,8 \\
\hline \multirow{7}{*}{ Antipsicótico } & Levomepromazina & 1 & 0,3 \\
\hline & Quetiapina & 2 & 0,6 \\
\hline & Decanoato de haloperidol & 5 & 1,5 \\
\hline & Risperidona & 116 & 36,3 \\
\hline & Olanzepina & 4 & 1,2 \\
\hline & Haldol & 2 & 0,6 \\
\hline & Cloridrato de Clorpromazina & 14 & 4,3 \\
\hline Estimulante & Metilfenidato & 48 & 15,8 \\
\hline
\end{tabular}




\section{CONCLUSÃO}

Mais da metade das crianças e adolescentes fazia uso de psicofármacos, em especial, antipsicóticos como a risperidona, o estimulante metilfenidato e os antidepressivos imipramina e fluoxetina. $\mathrm{O}$ sexo masculino era o que mais frequentavam o CAPSi. Alguns realizavam arteterapia e participavam de grupos terapêuticos, porém não apresentavam nenhum diagnóstico médico ou tinham como hipótese diagnóstica de TDAH e sintomas depressivos.

Tendo em vista que o comportamento infantil e adolescente não segue um padrão exato, é ne- cessário um trabalho multidisciplinar entre escola, família e profissionais da saúde para que tenham o cuidado dessas crianças e jovens não serem medicados sem a devida necessidade. Visto que, queixas comportamentais nem sempre são resolvidas com medicações, mas, sim, com encaminhamentos para oficinas e/ou terapias.

Diante do exposto e com a clara percepção da presença cada vez maior dos medicamentos no cotidiano da sociedade, sugerem-se novos estudos que acompanhem o paciente e sua evolução frente ao tratamento farmacológico realizado. Tal fato é importante para aprofundar o assunto e gerar dados que permitam compreender o entendimento da necessidade do tratamento medicamentoso.

\section{REFERÊNCIAS}

1. BRASIL. Lei $n^{\circ} 8.069$, de 13 de julho de 1990. Dispõe sobre o Estatuto da Criança e do Adolescente e dá outras providências. Diário Oficial da União; 1990.

2. WHO. World Health Organization. Physical status: use and interpretation of anthropometry. Geneva;1995.

3. Delvan JS, Portes JRM, Cunha MP, Menezes M, Legal, EJ. Crianças que utilizam os serviços de saúde mental: caracterização da população em uma cidade do sul do Brasil. Rev Bras Passeio Desenv Hum. 2010;20(2):228237.

4. Guerra AMC. A psicanálise no campo da Saúde Mental Infanto-Juvenil. Psychê. 2005;9(15):139-154.

5. Maiti R, Alloza J. Social Pharmacology: Expanding Horizons. Indian J Pharmacol. 2014;46(3):246-250. DOI: $10.4103 / 0253-7613.132151$

6. Bezerra IC, Jorge, MSB, Gondim APS, Lima LL de, Vasconcelos, MGF. "Fui lá no posto e o doutor me mandou foi pra cá": processo de medicamentalização e (des)caminhos para o cuidado em saúde mental na Atenção Primária. Interface - Com Saude Educ. 2014;18(48):61-74. DOI: $10.1590 / 1807-57622013.0650$

7. Rosa BPGD, Winograd M. Palavras e pílulas: sobre a medicamentalização do mal-estar psíquico na atualidade. Psicol Soc. 2011;23(spe):37-44.

8. Mills C. Psychotropic childhoods: global mental health and pharmaceutical children. Children Soc. 2014;28:194204. DOI: $10.1111 /$ chso. 12062

9. Baes CVW, Juruena, MF. Psicofarmacoterapia para o clínico geral. Rev Med. 2017;50(1):22-36. DOI: 10.11606/ issn.2176-7262.v50isup11.p22-36.
10. Ferreira, ACZ, Brusamarello T, Capistrano FC, Marin, MJS, Maftum MA. A vivência do portador de transtorno mental no uso de psicofármacos na perspectiva do pensamento complexo. Texto Contexto Enferm. 2017;26(3):01-10.

11. Thomas T, Stansifer L, Findling RL. Psicofarmacologia dos Transtornos Bipolares Pediátricos em Crianças e Adolescentes. Pediatric Clin N. 2011; 58(1):173-187.

12. Brzozowski FS, Caponi SNC. Medicalização dos Desvios de Comportamento na Infância: Aspectos Positivos e Negativos. Psicol Cienc Prof. 2013;33(1):208-221.

13. Couto MCV, Duarte CS, Delgado, PGG. A saúde mental infantil na Saúde Pública brasileira: situação atual e desafios. Braz J Psychiatr. 2008;30(4):390-398.

14. Schoen-Ferreira TH, Silva DA, Farias MA, Silvares EFM. Perfil e principais queixas dos clientes encaminhados ao centro de atendimento e apoio psicológico ao adolescente (CAAA) - UNIFESP/EPM. Psicol Estud. 2002;7(2):73-82.

15. Pais SC, Menezes I, Nunes JA. Saúde e escola: reflexões em torno da medicalização da educação. Cad Saude Publica. 2016;32(9):1-10. DOI: 10.1590/0102$311 \mathrm{X} 00166215$

16. Sanches VNL, Amarante PDC. Estudo sobre o processo de medicalização de crianças no campo da saúde mental. Saude Debate. 2014;38(102):506-514. DOI: 10.5935/0103-1104.20140047

17. Kamers M. A fabricação da loucura $n$ ainfância: psiquiatrização do discurso e medicalização da criança. Estilos Clin. 2013;18(1):153-165. 
18. Maturo A. Medicalization: current concept and future directions in a bionic society. Mens Sana Monogr. 2012;10:122-33. DOI: 10.4103/0973-1229.91587

19. Silva ACP, Luzio CA, Santos, KYP. A explosão do consumo de Ritalina. Rev Psicol (UNESP). 2012;11(2):4457.

20. Nascimento LYCMS, Rosa LS, Souza JC, Véras YA, Brêda MZ, Trindade RFC. Perfil de crianças e adolescentes acompanhados por um Centro de atenção psicossocial infanto-juvenil. Rev Enferm UFPE. 2014;8:1261-1272. DOI: 10.5205/reuol.5863-50531-1-ED.0805201421

21. Braghini S. Medicalização da infância: uma análise bibliográfica [Dissertação]. Campinas: Faculdade de Ciências Médicas. Universidade Estadual de Campinas Faculdade de Ciências Médicas. 2016.

22. Collares CAL, Moysés MAA. A educação na era dos transtornos. In: Viégas LS, Ribeiro MIS, Oliveira EC, Teles LL (Org.). Medicalização da educação e da sociedade: ciência ou mito? Salvador: EDUFBA, 2014.

23. Conrad P. The Shifting Engines of Medicalization. J Health Soc Behav. 2005;46(1):314. DOI: 10.1177/002214650504600102

24. Cordioli AV, Gallois CB, Isolan L. Psicofármacos: consulta rápida. 5nd. ed. Porto Alegre: Artmed. 2015.

25. Beltrame MM, Boarini ML. Saúde mental e infância: reflexões sobre a demanda escolar de um CAPSi. Psicol. Cienc. Prof. 2013,33(2):334-349.
26. Ignácio VTG, Nardi HC. A medicalização como estratégia biopolítica: um estudo sobre o consumo de psicofármacos no contexto de um pequeno município do Rio Grande do Sul. Acta Etho. 2007;19(3):88-95.

27. ONU. Organização das Nações Unidas. International Narcotics Control Board. Psychotropic substances. Statistics for 2008: assessments of annual medical and scientific requirement. New York; 2009.

28. Carvalho ILDN. Uso de psicofármacos em adolescentes atendidos pelos centros de atenção psicossocial infanto-juvenil de Fortaleza (CE) [Dissertação]. Fortaleza: Mestrado em Saúde Coletiva, Universidade de Fortaleza. 2012.

29. Pande MNR, Amarante PDC, Baptista TWF. Este ilustre desconhecido: Considerações sobre a prescrição de psicotrópicos na primeira infância. Cien Saude Colet [periódico na internet] (2018/Out). [Citado em 20/03/2019]. disponível em: http://www.cienciaesaudecoletiva.com. br/artigos/este-ilustre-desconhecido-consideracoes-sobre-a-prescricao-de-psicotropicos-na-primeira-infancia $/ 16968$ id $=16968$

30. Gorman JM. Treatment of generalized anxiety disorder. J Clin Psych. 2002;63(8):17-26.

31. Davidson JRT. Pharmacotherapy of generalized anxiety disorder. J Clin Psych. 2001;62(11):46-50.

32. Ramminger T, Brito, JC. "Cada CAPS é um CAPS": uma coanálise dos recursos, meios e normas presentes nas atividades dos trabalhadores de saúde mental. Psicol Soc. 2011;23(spe):150-160 